3 RIMOLDI, B.E., and LI, Q: 'Coded continuous phase modulation using ring convolutional codes', IEEE Trans., 1995, COM-43, (11), pp. $2714-2720$

4 AUlin, T., RYDBECK, N., and SUNDBERG, C.-E.W.: 'Continuous phase modulation - Part II: Partial response signalling', IEEE Trans., 1981, COM-29, (3), pp. 210-225

5 ANDERSON, J.B., AULIN, T., and SUNDberG, C.-E.: 'Digital phase modulation' (Plenum Press, New York, 1986)

\section{Hyper-resolution indoor channel impulse responses: Multipath components and $\boldsymbol{k}$ - factors}

\section{Ndzi, J. Austin and E. Vilar}

A hyper-resolution method has been used to estimate the wideband indoor channel impulse response at $1.3 \mathrm{GHz}$ in a variety of environments within a building. The number of multipath components $N$ and the Ricean $k$-factor have been investigated. The effect of the presence of people has also been monitored. The results show that if a $30 \mathrm{~dB}$ impulse response dynamic range threshold is considered, a value of $N$ less than 7 is applicable to all cases for $>90 \%$ of the time. It is also shown that the $k$-factor can be reliably estimated using only the three strongest components.

Introduction: Indoor wireless communications is rapidly expanding and has been boosted by the definition of the HIPERLAN standards in Europe and the de-regularisation of the ISM bands in the US. Present wireless indoor systems cannot meet the high data rate $(155 \mathrm{Mbit} / \mathrm{s})$ currently offered by wired networks due to multipath propagation and attenuation of transmitted signals [1]. To satisfy the demand for mobility and high data rates, some method of mitigating the effect of multipath must be implemented. One feasible solution is the use of smart antenna systems to select only the strongest component, given adequate time and angular resolution. To achieve this, knowledge of the number of multipath components $(N)$ reaching the receiver is of fundamental importance. In addition, the value of $N$ is important for the development of proper channel models.

Several investigations into the indoor impulse response have already been reported $[2,3]$. The fast Fourier transform based approach is widely used in data analysis but suffers from leakage which together with the assumption of periodicity, often gives a false impression of the existence of multipath components. Alternatively, high resolution parametric methods can be used. The results presented here have been computed using the singular value decomposition Prony algorithm [4, 5]. In this case the impulse response is estimated by fitting the measured channel transfer functions to a discrete non-periodic complex multipath model

$$
H(j \omega)=\sum_{i=1}^{N} a_{i} e^{-j \omega \tau_{i}}
$$

where $N$ is the number of paths (rays) and $a_{i}$ and $\tau_{i}$ are the complex amplitude and delay of the $i$ th multipath component, respectively. By using a suitably synthesised time domain signal, an impulse response resolution better than $5 \mathrm{~ns}$ has been achieved using a sounding spectrum $31.25 \mathrm{MHz}$ wide [6].

Measurement details: Using the channel sounder described in [6], measurements have been carried out in a large computer room (A), a long corridor (B) and in a small room (C). The ceiling in each location was $\sim 3 \mathrm{~m}$ above floor level and the volumes were $495.0,440.8$ and $151.3 \mathrm{~m}^{3}$ for locations A, B and C, respectively. The antennas were omni-directional half wavelength monopoles and the transmitted power was $14 \mathrm{dBm}$. The antenna heights were normally 2 and $1.6 \mathrm{~m}$ for the transmitter and receiver, respectively, and all measurements were LOS. Additional measurements were carried out in room A with and without the presence of people. In this case, an antenna separation of $14 \mathrm{~m}$ and heights of $1.6 \mathrm{~m}$ were used. The signal-to-noise ratio for all the measurements was $>$ $40 \mathrm{~dB}$.
Fig. 1 shows a data set which consists of 128 impulse responses estimated from transfer functions acquired over a period of $4 \mathrm{~s}$. The results shown were obtained with a person present in the vicinity of the receiver at an antenna separation of $1 \mathrm{~m}$. The Figure shows that there are three dominant paths and it can be seen that the signal suffers from attenuation and reflection as the person moves around. Two transfer functions and the corresponding impulse responses are illustrated in Fig. 2. The measured and fitted transfer functions are both given. The deep fade nulls are shown to be the result of the presence of a multipath component with magnitude equal or close to that of the strongest ray (snapshot 101 at 3.34s)

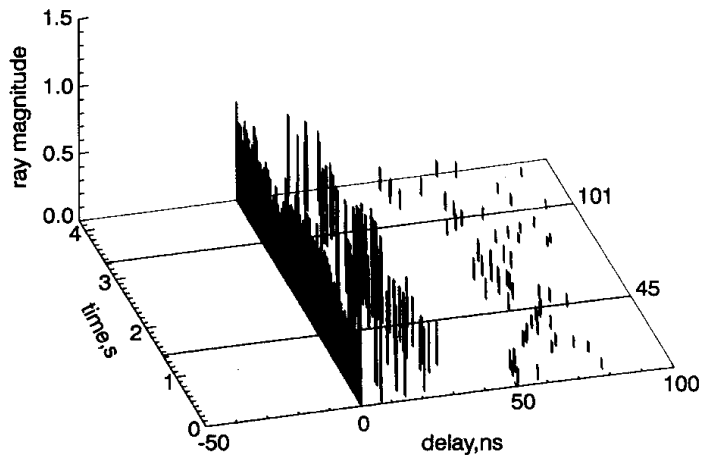

$145 / 1$

Fig. 1 Estimated channel impulse response illustrating variations in multipath due to person moving in vicinity of receiving antenna
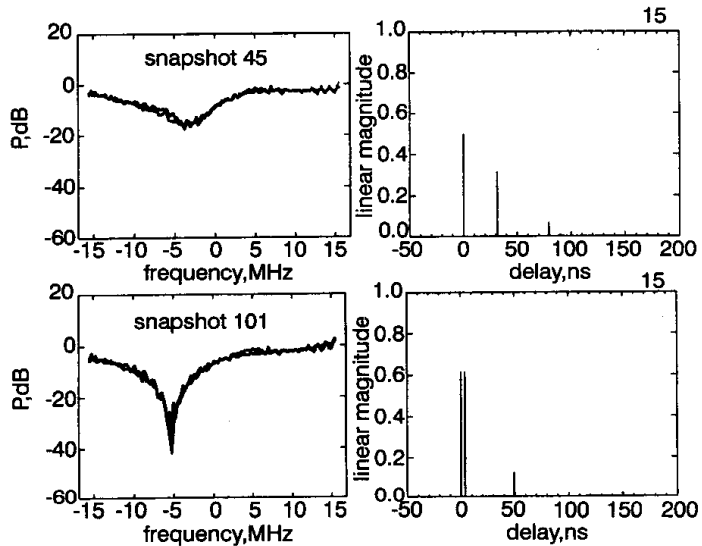

Fig. 2 Measured and fitted transfer functions and corresponding impulse responses

\section{measured}

— fitted

Number of multipath components $(N)$ : For each channel impulse response, the number of significant components $N$ above a given threshold was determined. Five thresholds were used and Fig. 3 illustrates typical cumulative distributions. As expected, as the threshold decreases more paths with small amplitudes are included. In general, the average value of $N$ increases with antenna separation for some thresholds, but the increase was not found to be as high as reported elsewhere [2].

The analysis of the data (see Fig. 3) shows that $N$ has a Gaussian distribution and that the fitting error decreases with the threshold as more echoes are considered. There is a large deviation between the cumulative distributions at $-30 \mathrm{~dB}$ and $-35 \mathrm{~dB}$ thresholds. At low signal-to-noise ratios, the large discrepancy suggests that most of the components are due to noise and thus not reliable. All results, including those not reported here, show that the $90 \%$ value of $N$ is always $<7$ for thresholds of $-30 \mathrm{~dB}$ and above.

$K$-factors: For each data set, the 128 impulse responses are superimposed with a bin size of 2 ns to obtain the delay power spectrum (DPS). Two types of factors, $k_{\text {scat }}$ and $k_{23}$, are used here. $k_{\text {scat }}$ is the 
logarithm of the ratio of the power in the bin containing the strongest path to the total scatter power in the DPS, assuming a threshold of $-30 \mathrm{~dB} . k_{23}$ is the logarithm of the ratio of the power in the main ray to the total power of the second and third rays for each impulse response, averaged over a data set. $k_{\text {scat }}$ is equivalent to the Ricean $k$-factor since all measurements were LOS.

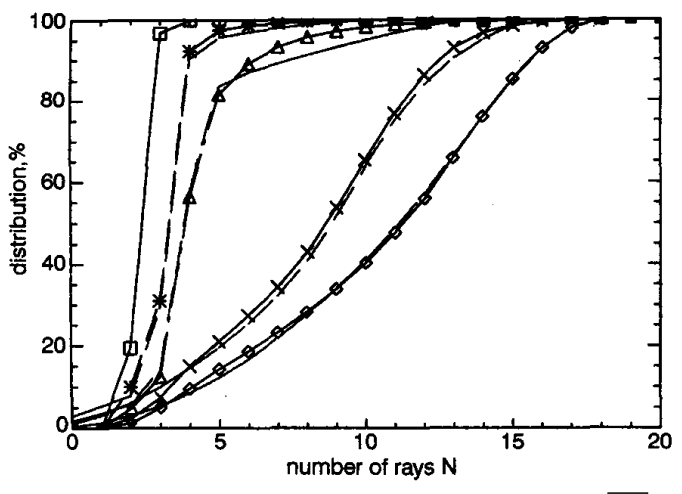

Fig. 3 Typical distribution of $N$ at different thresholds

Thresholds [dB]:

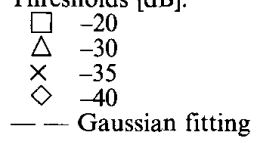

The statistics of $k$-factors for measurements in the three locations are summarised in Table 1. The mean values of $k_{\text {scat }}$ and $k_{23}$ are all $>6 \mathrm{~dB}$. All values of $k_{\text {scat }}$ can be reasonably estimated by $k_{23}$ for most LOS propagation conditions, especially over short distances. The results also show that $k$-factors generally decrease with antenna separation. This is consistent with the decrease in the power of the 'direct' component and an increase in scatter power.

Table 1: Mean $\mu$ and standard deviation $\sigma$ of $\mathrm{k}$-factor at different locations

\begin{tabular}{|c|cc|cc|}
\hline \multirow{2}{*}{ Location } & \multicolumn{2}{|c|}{$k_{23}[\mathrm{~dB}]$} & \multicolumn{2}{c|}{$k_{\text {scat }}[\mathrm{dB}]$} \\
\cline { 2 - 5 } & $\mu$ & $\sigma$ & $\mu$ & $\sigma$ \\
\hline Corridor & 6.7 & 3.0 & 6.3 & 4.0 \\
\hline Room A & 6.6 & 4.5 & 7.0 & 4.9 \\
\hline Room C & 7.5 & 3.7 & 7.2 & 3.7 \\
\hline
\end{tabular}

Effect of people on $N$ and $k$-factor: Two data blocks, containing 150000 impulse responses each, were acquired in room A (a large computer room). They were obtained over time periods of $12 \mathrm{~h}$, with and without people in the room. The results showed that the presence of people resulted in a $2 \mathrm{~dB}$ attenuation of the mean signal level with the mean value of $N$ decreasing from 4 to 3 for thresholds $<25 \mathrm{~dB}$. The $k$-factors, $k_{\text {scat }}$ and $k_{23}$ were also found to have decreased by 2.5 and $4.5 \mathrm{~dB}$, respectively. This indicates that the direct path was attenuated and that the echos were selectively attenuated or reflected away from the receiver. The standard deviation of $k_{\text {scat }}$ and $k_{23}$ increased by factors $>5$, indicating that the channel response varied widely in the presence of people.

Conclusions: Hyper-resolution impulse responses from three locations within a building have shown that the number of multipath components with amplitudes within $30 \mathrm{~dB}$ of the strongest path is $<7$ for $90 \%$ of the time. The results also show that the channel Ricean $k$-factor can be reasonably estimated using only the three strongest components. The presence of people in the channel introduced very significant variations in the impulse response and in general, the mean values of both the $k$-factor and $N$ decreases when people are walking in the proximity of the channel.

Acknowledgments: Sponsorship of the development of the channel sounder by the UK Radiocommunications Agency, is gratefully acknowledged.
(C) IEE 1999

Electronics Letters Online No: 19990491

DOI: 10.1049/el:19990491

D. Ndzi, J. Austin and E. Vilar (Microwave Telecommunications and Signal Processing Research Group, Department of Electrical and Electronic Engineering, University of Portsmouth, Anglesea Road, Portsmouth PO1 3DJ, United Kingdom)

E-mail: dln@ee.port.ac.uk

\section{References}

1 HASHEMI, H.: 'The indoor radio propagation channel', Proc. IEEE, 1993, 81, (7), pp. 943-968

2 HASHEMI, H: 'Impulse response modeling of indoor radio propagation channels', IEEE J. Sel. Areas Commun., 1993, SAC11, (7), pp. 967-978

3 RAPPAPORT, T.S.: 'Statistical channel impulse response models for factory and open plan building radio communication system design', IEEE Trans., 1991, COM-39, (5), pp. 794-807

4 NDZI D. AUSTIN, J., and VILAR, E.: 'Statistics of coherence bandwidth and delay spread on an over-the-sea transhorizon path'. Proc. Int. Conf. Telecommunications (ICT'98), Porto Carras, Greece, June 1998, Vol. 1, pp. 100-104

5 LAU, W.H., AUSTIN, J., HEWITT, A., VILAR, E., and MARTIN, L: 'Analysis of the time-variant structure of the microwave line-ofsight multipath phenomena', IEEE Trans., 1991, COM-39, (6), pp. $847-855$

6 AUSTIN, J., DITMAR, W.P.A., LAM, W.K., VILAR, E., and WAN, K.W.: 'A spread spectrum communications channel sounder', IEEE Trans., 1997, COM-45, (7), pp. 840-847

\section{Outer-loop control of target SIR for fast transmit power control in turbo-coded W-CDMA mobile radio}

\author{
H. Kawai, H. Suda and F. Adachi
}

A technique for obtaining outer-loop control of the target SIR for closed-loop fast transmit power control (TPC) is presented for turbo-coded wideband DS-CDMA (W-CDMA) mobile radio systems. The frame error rate (FER) of an intermediate decoding output in the turbo-decoder, i.e. before the final iteration stage is reached, is measured to control the target SIR. The bit error rate (BER) after the final decoding iteration can be maintained accurately at the prescribed $B E R$ value, e.g. $B E R=10^{-6}$, without losing the tracking ability in the presence of slowly changing propagation channel conditions. Computer simulation results supporting this ability are reported.

Introduction: In DS-CDMA mobile radio, severe multiple access interference (MAI) is produced due to distance-dependent path losses (the well-known 'near-far problem'), slow random pathlosses (shadowing), and fast multipath fading. A closed-loop fast transmit power control (TPC) technique based on the measurement of the instantaneous signal-to-average interference plus background noise ratio (SIR) is indispensable for minimising the adverse effects of MAI [1]. In closed-loop fast TPC, a base station receiver measures the received SIR of the signal transmitted from a given mobile station, compares it to a prescribed target SIR, and then generates a binary TPC command to raise or lower the mobile transmit power in order to maintain the received bit error rate (BER) at the required value. However, the target SIR must be different for different propagation channel conditions, such as the power delay profile, the number of resolvable propagation paths, the fading maximum Doppler frequency (the velocity of the mobile terminal), etc. Therefore, the target SIR must be adjusted by introducing outer-loop control to track slowly changing channel conditions [1]. Because in general it is difficult to measure the $B E R$, a frame error rate (FER) measurement using cyclic redundancy checks (CRCs) is often used in outer-loop control. However a problem may occur when a very low BER is desired for data transmission, e.g. BER $=10^{-6}$ as required in IMT-2000 [2]. Since the corresponding FER is also low, a fairly long FER measurement interval is required. For example, the FER value corresponding to $\mathrm{BER}=10^{-6}$ is $<10^{-3}$ for a transmission rate of $32 \mathrm{kbit} / \mathrm{s}$ and 